\title{
Spatiotemporal neuromodulation therapies engaging muscle synergies improve motor control after spinal cord injury
}

\author{
Nikolaus Wenger ${ }^{1-3,17}$, Eduardo Martin Moraud ${ }^{4,17}$, Jerome Gandar ${ }^{1,17}$, Pavel Musienko ${ }^{1,5-7}$, Marco Capogrosso ${ }^{4,8}$, \\ Laetitia Baud $^{1}$, Camille G Le Goff ${ }^{1}$, Quentin Barraud ${ }^{1}$, Natalia Pavlova ${ }^{1,5}$, Nadia Dominici ${ }^{1,9}$, Ivan R Minev ${ }^{10}$, \\ Leonie Asboth $^{1}$, Arthur Hirsch ${ }^{10}$, Simone Duis ${ }^{1}$, Julie Kreider ${ }^{1}$, Andrea Mortera ${ }^{4}$, Oliver Haverbeck ${ }^{11}$, \\ Silvio Kraus ${ }^{11}$, Felix Schmitz ${ }^{12}$, Jack DiGiovanna ${ }^{4}$, Rubia van den Brand ${ }^{1}$, Jocelyne Bloch ${ }^{13}$, Peter Detemple ${ }^{12}$, \\ Stéphanie P Lacour ${ }^{10}$, Erwan Bézard ${ }^{14-16}$, Silvestro Micera ${ }^{4,8} \&$ Grégoire Courtine $^{1,13}$
}

\begin{abstract}
Electrical neuromodulation of lumbar segments improves motor control after spinal cord injury in animal models and humans. However, the physiological principles underlying the effect of this intervention remain poorly understood, which has limited the therapeutic approach to continuous stimulation applied to restricted spinal cord locations. Here we developed stimulation protocols that reproduce the natural dynamics of motoneuron activation during locomotion. For this, we computed the spatiotemporal activation pattern of muscle synergies during locomotion in healthy rats. Computer simulations identified optimal electrode locations to target each synergy through the recruitment of proprioceptive feedback circuits. This framework steered the design of spatially selective spinal implants and real-time control software that modulate extensor and flexor synergies with precise temporal resolution. Spatiotemporal neuromodulation therapies improved gait quality, weight-bearing capacity, endurance and skilled locomotion in several rodent models of spinal cord injury. These new concepts are directly translatable to strategies to improve motor control in humans.
\end{abstract}

Spinal cord injury (SCI) disrupts communication between supraspinal centers and spinal circuits, which leads to a range of motor disabilities. Neuromodulation strategies provide access to surviving circuits and pathways to alleviate these deficits ${ }^{1,2}$. In particular, electrical and chemical neuromodulation of the lumbar spinal cord mediates significant improvement of lower-limb motor control in animal models ${ }^{3-7}$ and humans ${ }^{6,8-11}$ with SCI.

Computer simulations ${ }^{12-14}$ and experimental studies ${ }^{7,15-17}$ have provided evidence that electrical neuromodulation applied to the dorsal aspect of lumbar segments primarily engages proprioceptive feedback circuits. The prevailing view is that the recruitment of these neural pathways activates central pattern-generating networks ${ }^{9,18}$ and raises the excitability of spinal circuits to a level that enables sensory information to become a source of motor control ${ }^{19}$. This framework enacts two fundamental limitations in the clinical application of electrical neuromodulation therapies. First, the spatial location of stimulation is confined to single spinal cord regions that are selected on the basis of empirical mapping experiments ${ }^{9,20}$. Second, the temporal structure of stimulation protocols is restricted to nonmodulated patterns that remain constant during motor execution, regardless of the current state of lower-limb movements ${ }^{8,9,15}$.

The production of lower-limb movements involves activation of spatially distributed motoneurons ${ }^{21,22}$ following precise temporal sequences ${ }^{23,24}$ that are continuously adjusted through sensory feedback ${ }^{25}$. Current stimulation protocols do not attempt to reproduce these spatiotemporal patterns of motoneuron activation. We hypothesized that electrical neuromodulation therapies integrating spatial selectivity and temporal structure matching the natural dynamics of motoneuron activation would improve stimulation efficacy and enhance the quality and vigor of lower-limb movements after SCI.

To test this hypothesis, we first conducted anatomical and functional experiments to visualize the spatiotemporal pattern of hindlimb motoneuron activation in healthy rats. We found that walking involves

\footnotetext{
${ }^{1}$ International Paraplegic Foundation Chair in Spinal Cord Repair, Center for Neuroprosthetics and Brain Mind Institute, School of Life Sciences, Swiss Federal Institute of Technology (EPFL), Lausanne, Switzerland. 2Department of Neurology with Experimental Neurology, Charité-Universitätsmedizin Berlin, Berlin, Germany. ${ }^{3}$ Center for Stroke Research Berlin, Charité-Universitätsmedizin Berlin, Berlin, Germany. ${ }^{4}$ Bertarelli Foundation Chair in Translational Neuroengineering, Center for Neuroprosthetics and Institute of Bioengineering, School of Bioengineering, EPFL, Lausanne, Switzerland. ${ }^{5}$ Motor Physiology Laboratory, Pavlov Institute of Physiology, St. Petersburg, Russia. ${ }^{6}$ Laboratory of Neuroprosthetics, Institute of Translational Biomedicine, St. Petersburg State University, St. Petersburg, Russia. ${ }^{7}$ Lab of Neurophysiology and Experimental Neurorehabilitation, Children's Surgery and Orthopedic Clinic, Department of Nonpulmonary Tuberculosis, Institute of Physiopulmonology, St. Petersburg, Russia. ${ }^{8}$ The BioRobotics Institute, Scuola Superiore Sant'Anna, Pisa, Italy. ${ }^{9}$ MOVE Research Institute Amsterdam, Faculty of Behavioural and Movement Sciences, VU University Amsterdam, Amsterdam, the Netherlands. ${ }^{10}$ Bertarelli Foundation Chair in Neuroprosthetic Technology, Center for Neuroprosthetics and Institute of Bioengineering, EPFL, Lausanne, Switzerland. ${ }^{11}$ Micromotive GmbH, Mainz, Germany. ${ }^{12}$ Fraunhofer Institute for Chemical Technology-Mainz Institute for Microtechnology (ICT-IMM), Mainz, Germany. ${ }^{13}$ Centre Hospitalier Universitaire Vaudois (CHUV), Lausanne, Switzerland. ${ }^{14}$ Motac Neuroscience Inc., Beijing, China. ${ }^{15}$ University of Bordeaux, Institut des Maladies Neurodégénératives, Bordeaux, France. ${ }^{16} \mathrm{CNRS}$, Institut des Maladies Neurodégénératives, Bordeaux, France. ${ }^{17}$ These authors contributed equally to this work. Correspondence should be addressed to G.C. (gregoire.courtine@epfl.ch).
} 
the alternating activation of spatially restricted hot spots underlying extensor and flexor muscle synergies. To access these hot spots, we developed neuromodulation strategies to specifically target proprioceptive feedback circuits in the dorsal roots. We used computer simulations to determine the optimal electrode locations for recruiting specific subsets of dorsal roots. The results of these simulations steered the design of spatially selective spinal implants and real-time control software to modulate extensor and flexor muscle synergies with precise temporal resolution, adjusted through movement feedback. Spatiotemporal neuromodulation therapies reinforced extension and flexion components for each hindlimb independently, which improved a range of important gait features after complete SCI. This conceptually new stimulation strategy improved dynamic balance and skilled descending control during quadrupedal locomotion in rats with clinically relevant SCI.

\section{RESULTS}

\section{Dynamics of hindlimb motoneuron activation during gait}

We first injected pairs of antagonist muscles spanning each hindlimb joint with Fluorogold to visualize the spatial location of hindlimb motoneurons. To combine tracings from multiple rats, we elaborated a three-dimensional (3D) digital model of lumbar segments using retrogradely labeled tibialis anterior motoneurons as a landmark (Fig. 1a).
As expected, motoneurons were segregated into well-defined columns of motor pools innervating a single muscle. Each column occupied a reproducible spatial location that created a rostrocaudal gradient of flexor and extensor motoneurons innervating proximal to distal hindlimb muscles (Supplementary Fig. 1a).

We then implanted bipolar electrodes into hindlimb muscles of seven intact rats to record simultaneously the electromyographic activity of all traced muscles during locomotion (Fig. 1b). We projected the recorded muscle activity onto motoneuron locations to visualize spatiotemporal maps of motoneuron activation (Fig. 1c). These maps, which were consistent across rats (Supplementary Fig. 2a), revealed that locomotion involves a burst of motoneuron activity in the fourth lumbar segment (L4) to L6 during stance and a burst of motoneuron activity centered mostly around segments L2-L4 during swing. Gaussian clustering analysis identified these two spatial locations $(P<0.01)$, which we termed hot spots (Fig. 1c).

To identify the functionality and temporal structure underlying the activation of these hot spots, we decomposed muscle activity into functional modules termed muscle synergies ${ }^{24,26}$ using non-negative matrix factorization ${ }^{27}$. Four temporal activation profiles coalescing weighted combinations of the recorded muscle activity were sufficient to reconstruct more than $92 \%$ of the variance in the original signals $(92.0 \pm 1.1$, s.e.m.) (Fig. 1d). To link muscle synergies to the activation
Figure 1 Spatiotemporal activation of hindlimb motoneuron during locomotion. (a) Tracer injections into the tibialis anterior (TA) muscle to label motoneurons. Top-down and coronal snapshots of 3D lumbosacral reconstructions; each TA motoneuron is represented by a single dot. The same procedure was applied to gluteus medius (GM), iliopsoas (IL), vastus lateralis (VL), semitendinosus ( $\mathrm{St}$ ), biceps femoris (BF), gastrocnemius medialis (MG), gastrocnemius lateralis (LG), extensor digitorum longus (EDL) and flexor hallucis longus (FHL) muscles. The rostrocaudal location and center (white square) of each reconstructed motoneuron column is indicated in red or blue for muscles acting functionally for extension or flexion, respectively. (b) Muscle activity during locomotion in an intact rat. (c) Muscle activity projected onto the motoneuron location matrix to elaborate the spatiotemporal map of motoneuron activation (left); the spatially restricted hot spots emerging during gait were extracted by applying a Gaussian cluster algorithm onto the map (right). (d) Mean (thick lines) and individual (thin lines, $n=7$ rats) temporal activation profiles of muscle synergies (Syn1-Syn4) (left) and histogram plots (right) reporting muscle weights (arbitrary units (a.u.)) for each rat (vertical bars) and muscle type on each muscle synergy. (e) Spatiotemporal maps of muscle synergy activation, elaborated by multiplying each synergy with its corresponding weighted motoneuron matrix. (f) Model of spinal segments showing the temporal sequence underlying the recruitment of muscle synergies and the corresponding activation of extensor and flexor hot spots. (g) Illustration of the timing underlying muscle synergy activation, as captured in the spatiotemporal trajectory of the hindlimb endpoint. a
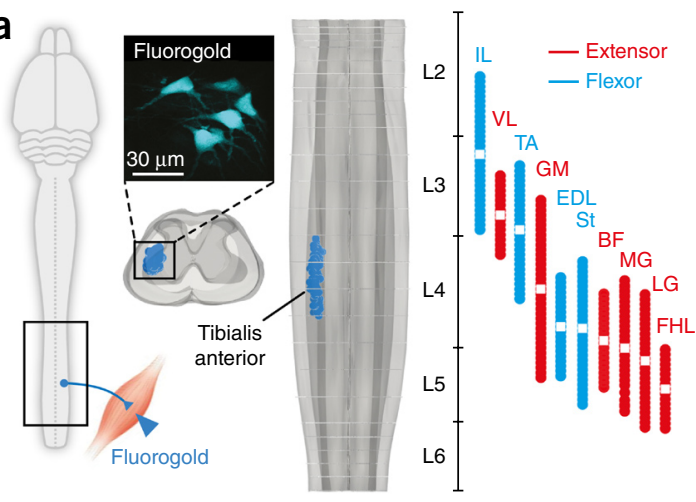

b
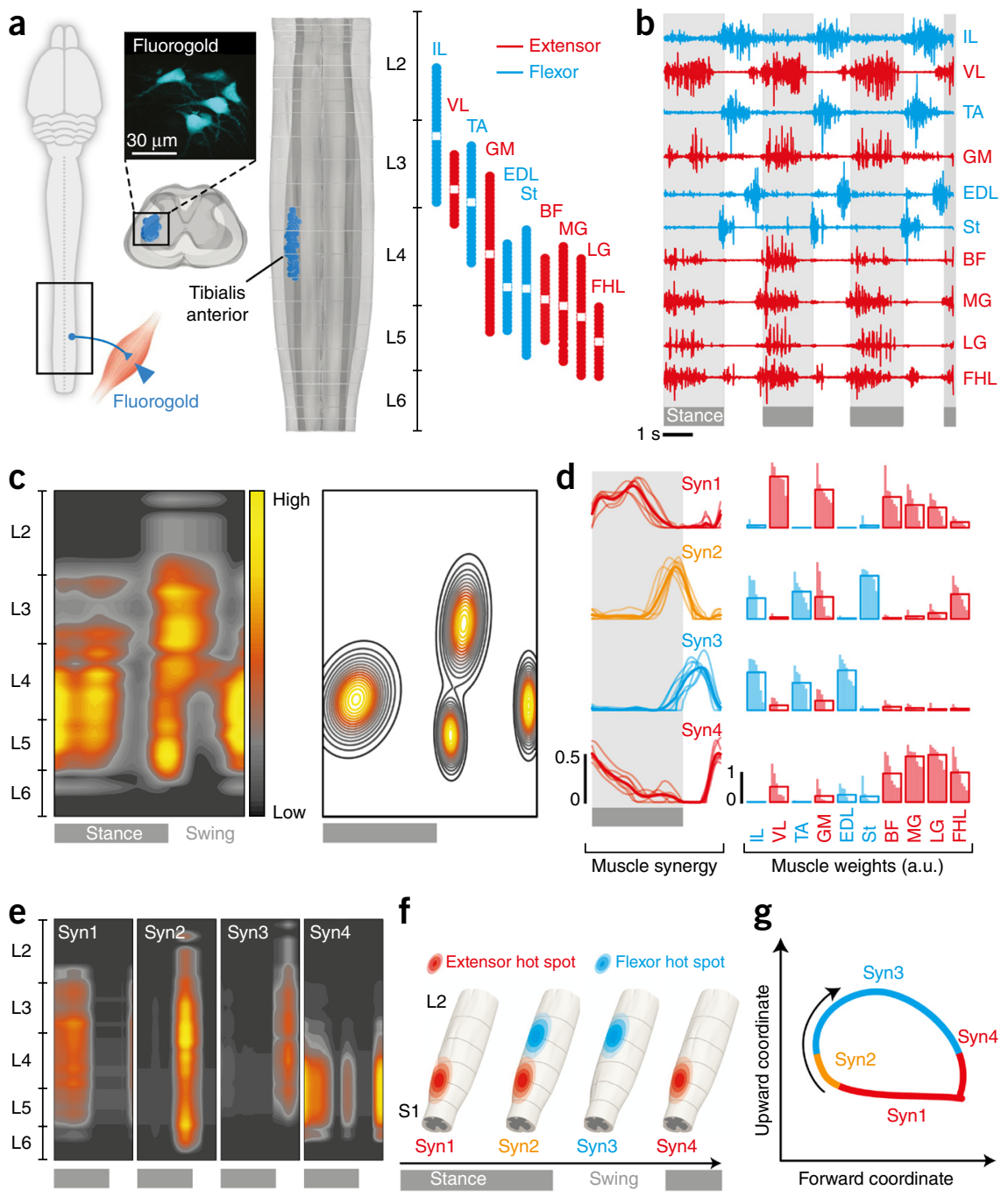
of hot spots, we extracted the spatiotemporal map of motoneuron activation for each synergy independently (Fig. 1e). This analysis revealed that the first and fourth synergies produced hindlimb extension and enabled resistance to gravity through activation of the first hot spot, which we termed the extensor hot spot. These synergies were activated during stance and before foot strike. The third synergy promoted hindlimb flexion during swing by activation of the second, or flexor, hot spot. The second synergy emerged during the transition from stance to swing and involved simultaneous recruitment of both hot spots. These experiments thus uncovered the function and temporal structure underlying the successive activation of spatially restricted extensor and flexor hot spots during locomotion (Fig. 1f). The spatiotemporal trajectory of the hindlimb foot (hindlimb endpoint) accurately captured this pattern (Fig. 1g).

\section{Electrode position to target extensor or flexor hot spots}

Epidural electrical stimulation of lumbar segments primarily recruits large-diameter proprioceptive fibers in the dorsal roots ${ }^{7,12,28,29}$. We thus hypothesized that electrodes targeting specific subsets of dorsal roots might provide access to spatially restricted spinal segments associated with extensor or flexor hot spots.

We reconstructed the 3D location of dorsal roots innervating each lumbar segment (Supplementary Fig. 1b-c) and integrated these anatomical data into an experimentally validated computational model of epidural electrical stimulation ${ }^{12}$. We then ran computer simulations using an optimization function that searched optimal electrode locations to recruit the dorsal roots projecting to spinal segments containing extensor or flexor hot spots on each side of the spinal cord (Supplementary Fig. 3). Simulations revealed that electrodes located $1.00 \mathrm{~mm}$ laterally at the border between segments L2 and L3, and $0.75 \mathrm{~mm}$ laterally at segment $\mathrm{S} 1$, preferentially recruited the dorsal roots projecting to flexor and extensor hot spots, respectively (Fig. 2a,b).

To verify these predictions, we delivered stimulation at the identified electrode locations during acute experiments. Simultaneous electromyographic recordings of mono- and polysynaptic motor responses in hindlimb muscles enabled the reconstruction of spinal segment activation for each site of stimulation. These experiments confirmed the ability to target the spatially restricted hot spots unilaterally using electrode locations identified with computer simulations (Fig. 2c,d).

\section{Hardware: spatially selective spinal implants}

We exploited microfabrication techniques to design spinal implants integrating the electrode configuration identified with computer simulations (Supplementary Fig. 4a). The topologies and dimensions of the implants were tailored to the anatomy of the spinal cord and vertebra structures (Supplementary Fig. 1c).

We developed surgical procedures, including a tailored fixation structure, for placing and stabilizing spinal implants overlying the targeted epidural spinal cord regions (Fig. 2e and Supplementary Fig. 4d). Repeated impedance and electrophysiological evaluations over 6 weeks (Supplementary Fig. 5a-b) and the absence of inflammatory response after explantation (Supplementary Fig. 5c-e) verified the long-term functionality and bio-integration of the spinal implants.

Delivery of short bursts of electrical stimulation (four pulses at $40 \mathrm{~Hz}$ ) through the spinal implants elicited the predicted unilateral movements of extension or flexion during suspended conditions in six intact rats (Fig. 2f and Supplementary Video $\mathbf{1}$ ).

Software: adjustment of parameters through movement feedback We next exploited an advanced real-time control platform ${ }^{7}$ to implement spatiotemporal neuromodulation therapies. We elaborated signal-processing algorithms that detect the timing of gait events on the basis of real-time tracking of bilateral hindlimb kinematics (Fig. 3). The continuous temporal sequence of gait events was derived from the angular displacement of the hindlimb endpoint around its center of rotation (Fig. 3). This progression of the foot in space resembles a clock hand that shows the current timing of gait (Fig. 1g).

A finite-state machine logic triggered the activation of each electrode when the hindlimb endpoint trajectory crossed a user-defined
Figure 2 Design, fabrication and validation of spatially selective spinal implants.

(a) Left, Gaussian curves of spatial distribution of flexor or extensor hot spots for which an optimization algorithm identifies the cost of preferentially activating dorsal roots projecting to each. Middle, 3D reconstruction of dorsal roots ( $n=3$ rats). Right, computer simulations iterated over a grid of electrodes covering the targeted neural structures. Black circles indicate the optimal locations of electrodes.

(b) Computer simulations showing isopotential 1 -V surface after a $150-\mu$ A stimulation at each identified electrode location, including the resulting dorsal root activation. $(\mathbf{c}, \mathbf{d})$ Spatial maps of spinal segment activation for the optimal electrodes predicted by computer simulations (c) and experimental validation in anesthetized rats $(d)(n=4)$. The map is computed from motor responses recorded from eight hindlimb muscles as in Figure 1a. (e) Top, photographs of spinal implant (left), connector (right) and electrodes (inset). Bottom, 3D rendering reconstructed from high-resolution microcomputed tomography scans done 5 weeks after implantation. (f) Stick diagram decomposition of hindlimb movements for the ipsilateral (color) and contralateral (black) side to the stimulation with a series of four bursts $(40 \mathrm{~Hz}$ ) delivered through the electrodes targeting each hot spot under suspended conditions (left) and histogram plots (right) reporting the vertical displacement of the hindlimb endpoint. ${ }^{*} P<0.05 ;{ }^{* *} P<0.01 ;{ }^{* *} P<0.001$ one-way ANOVA; $n=6$, mean \pm s.e.m. b

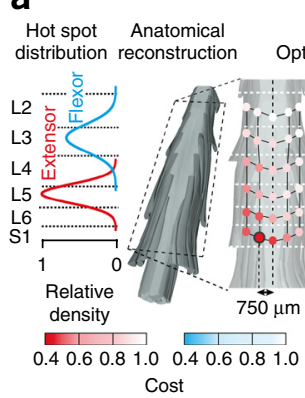

C
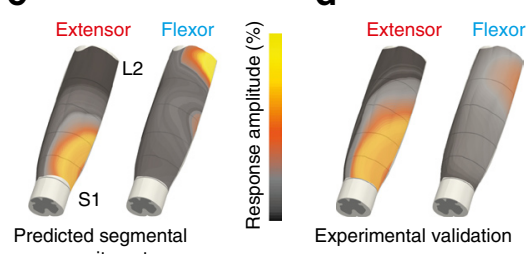

\section{Optimal site}

e

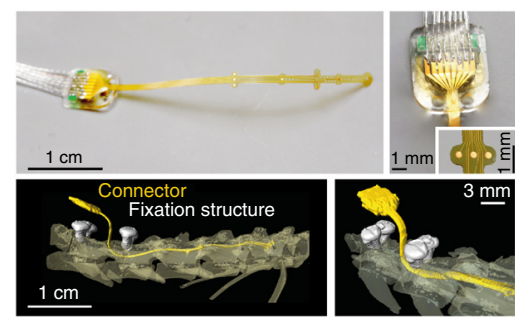

$\square$ Gray matter $\square$ Cerebrospinal fluid

f Stimulation of Stimulation of flexor hot spot extensor hot spot Ipsi Contra

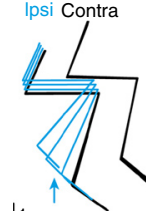
Ipsi Contra

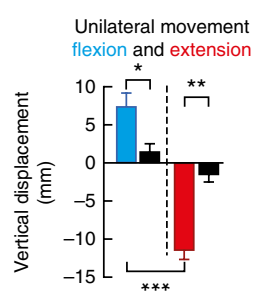


Figure 3 Software to adjust spatiotemporal neuromodulation in real-time during locomotion. Computational platform to trigger adjustments of the temporal structure, spatial configuration and stimulation parameters of the neuromodulation therapies. Rats are supported bipedally in a robotic system provided vertical support during locomotion on a motorized treadmill belt (top left). A high-resolution video system allows realtime monitoring of the left and right hindlimb endpoints. The angular displacements of hindlimb endpoints around a calculated center of rotation are converted into angular coordinates (dotted gray lines, top right). The on and off states of electrodes targeting extensor- and flexorrelated hot spots are triggered when the angular coordinates cross user-defined thresholds customized for each rat. The stimulation profile module enables tuning the amplitude and frequency of stimulation on the basis of the therapist or control policies. The diagram represents the relationship between the vertical displacement of the foot and the activation of extensor and flexor hot spot and how the spatially selective electrodes are turned on and off to replicate this activation pattern.

angular threshold (Fig. 3 and Supplementary Video 2). Adjustments to amplitude or frequency could be tuned on the basis of control policies $^{7}$ (Fig. 3). The time necessary to trigger stimulation on the basis of movement feedback remained below $20 \mathrm{~ms}$, which is 40 times faster than the average gait cycle duration in rats ( $800 \pm 146 \mathrm{~ms}$, s.d.). Stimulation was triggered with $97.8 \%$ accuracy for a temporal window constrained within less than $5 \%$ of the relative gait cycle duration ( $n=140$ gait cycles).

This computational infrastructure provides the technological framework for real-time adjustment of neuromodulation parameters over multiple electrodes on the basis of high-fidelity kinematic feedback.

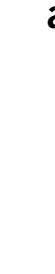

a
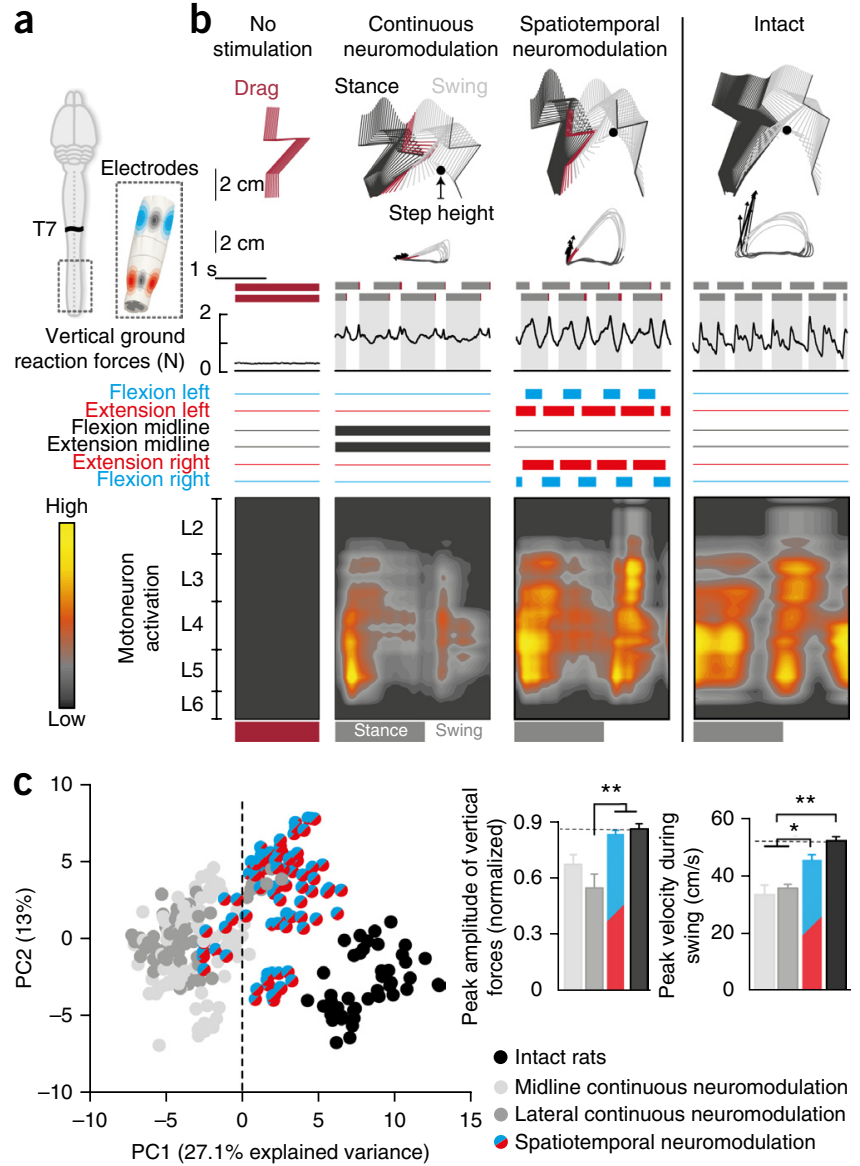

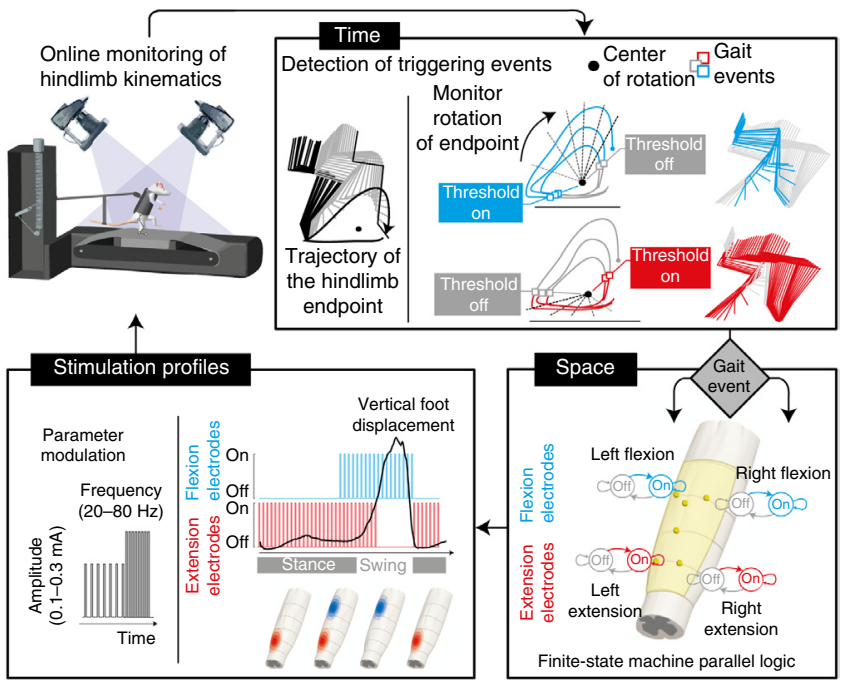

\section{Spatiotemporal neuromodulation after complete $\mathbf{S C I}$}

We next used this hardware and software to test whether spatiotemporal neuromodulation therapies mediate gait improvements better than continuous stimulation protocols after SCI. Adult rats were subject to SCI via a complete transection of thoracic segment T7 (Fig. 4a). We inserted the tailored spinal implant during the same surgery. Five weeks after SCI, all rats showed complete hindlimb paralysis when positioned bipedally in a robotic body weight support system ${ }^{4}$ (Fig. 4 b). Ten minutes before testing, we administered a serotoninergic replacement therapy ${ }^{4,5}$ that compensates for the interrupted source of monoaminergic modulation after injury. Continuous electrical neuromodulation delivered over the midline of segments L2-L3 and S1 enabled all rats tested to perform coordinated locomotion in response to treadmill motion, as previously reported ${ }^{4}$ (Fig. 4 b). However, the spatiotemporal maps of motoneuron activation differed from those observed in intact rats (Fig. 4b); vertical ground reaction forces were lower $(P<0.01)$ and foot movement during swing was more limited $(P<0.01)$ (Fig. 4b) in injured rats than intact rats.

To optimize the temporal structure of spatiotemporal neuromodulation therapies, we evaluated the impact of varying the time of onset or end of stimulation. Adjustment of the activation timing for the

Figure 4 Spatiotemporal neuromodulation reproduces the natural pattern of motoneuron activation. (a) Rats received complete $\mathrm{SCl}$ at T7 and a spinal implant with conventional midline electrodes (black) and spatially selective lateral electrodes (blue and red). (b) Locomotion in rats on a treadmill without stimulation and with continuous neuromodulation applied over the midline of lumbar and sacral segments and during spatiotemporal neuromodulation. For each condition (same rat, $n=5$ ) and intact rats $(n=3)$, a stick diagram decomposition of left hindlimb movement is shown together with successive trajectories of the hindlimb endpoint, the velocity and orientation of the foot trajectory at toe off (vector with arrowheads); stance (dark gray), drag (dark red) and swing phases (light gray) of both hindlimbs; and vertical ground reaction forces during a continuous sequence of steps. The horizontal bars (blue, red, black) indicate the current state of the electrodes. The corresponding spatiotemporal maps of motoneuron activation were calculated over ten consecutive steps. Conventions are as in Figure 1. (c) Left, PC analysis applied on 137 gait parameters with all the gait cycles recorded in rats $(n=5)$ under different conditions of neuromodulation, represented in a PC space. Right, histogram plots of peak amplitude of vertical ground reaction forces and peak velocity of the foot during swing for different neuromodulation conditions and for intact rats. ${ }^{*} P<0.05$; ${ }^{* *} P<0.01$, Kruskal-Wallis test; data are mean and s.e.m. 
electrode targeting the flexor hot spot led to a gradual tuning of flexion-related variables such as flexor muscle activity, step height and foot acceleration during swing (Supplementary Fig. 6). We detected similar modulation for extensor-related variables, such as extensor muscle activity and vertical ground reaction force, when varying the activation timing of the electrode targeting the extensor hot spot (Supplementary Fig. 6). The concurrent activation of flexor and extensor hot spots at the end of stance was also crucial to prevent foot dragging (Supplementary Fig. 6). For each electrode, we selected the timing that yielded optimal values for this ensemble of gait parameters. The resulting temporal structure matched the temporal activation profiles of muscle synergies exposed in intact rats (Supplementary Fig. 6c).

We tested whether this optimized stimulation protocol was capable of reproducing the spatiotemporal maps of motoneuron activation underlying locomotion of intact rats. In all rats tested, spatiotemporal neuromodulation therapies reinforced the amplitude of electromyographic signals and improved activation timing in nearly all recorded hindlimb muscles (Supplementary Fig. 7). This modulation yielded spatiotemporal maps of hindlimb motoneuron activation that resembled those observed in intact rats (Fig. 4b, Supplementary Fig. 2 and Supplementary Video 2).

Finally, we compared the improvements in locomotion after spatiotemporal neuromodulation to those after continuous neuromodulation. To quantify gait performance, we computed numerous parameters from recordings of kinematic, kinetic and muscle activity ( $n$ =137) (Supplementary Table 1). To weight their relative importance objectively, we subjected the parameters to a principal component (PC) analysis ${ }^{4,5}$. We visualized gait patterns in the new space created by $\mathrm{PC} 1$ and PC2, where PC1 explained the highest amount of variance (27\%) and reflected the degree of similarity to intact rats (Fig. 4c). Analysis of scores on PC1 revealed that gait patterns were closer to those of intact rats after spatiotemporal neuromodulation than after continuous neuromodulation $(P<0.05)$ (Supplementary Fig. 8). We extracted parameters that highly correlated (factor loading $>|0.5|$ ) with $\mathrm{PC} 1$ and regrouped them into functional clusters corresponding to basic movement features. This analysis revealed that spatiotemporal neuromodulation normalized many key features of hindlimb movements (41 of 137 significantly improved parameters, $P<0.05$ ) (Supplementary Fig. 8). Continuous stimulation delivered through the four spatially selective lateral electrodes did not improve gait performance $(P<0.05)$ (Fig. 4c and Supplementary Fig. 8), confirming the synergy between spatial selectivity and temporal structure.

\section{Gradual adjustment of extension and flexion movements}

Modulation of stimulation amplitude and frequency leads to gradual adjustments of hindlimb movements ${ }^{7}$. We asked whether this modulation could be superimposed onto spatiotemporal neuromodulation therapies in injured rats. We first varied the amplitude of stimulation delivered through one of the spatially selective lateral electrodes. A progressive increase of stimulation amplitude for the electrode targeting extensor or flexor hot spots promoted a selective and gradual augmentation of extensor or flexor muscle activity on the stimulation side in all rats tested $(n=5, P<0.05)$ (Fig. 5a). This tuning translated into an incremental enhancement of extension and flexion components (Fig. 5b), which supported the control of hindlimb extension during stance and foot trajectories during swing over ranges that reached the anatomical limits of motion (Supplementary Video 2). In contrast, increases in stimulation amplitude blocked hindlimb movements when stimulation was delivered without temporal structure (Supplementary Video 2). Muscle activity resulted from a succession of motor responses elicited after each pulse of stimulation (Supplementary Fig. 9), which probably originated from the recruitment of proprioceptive feedback circuits ${ }^{7,12,28,29}$. Increases in stimulation amplitude led to commensurate augmentation of the amplitude of these responses (Supplementary Fig. 9).

We then verified that the previously observed modulation of step height with adjustment of stimulation frequency ${ }^{7}$ was preserved
Figure 5 Selective and gradual modulation of extension and flexion components.

(a) Locomotor sequences recorded during spatiotemporal neuromodulation in an injured rat with different stimulation amplitudes for the electrode targeting the extensor (left) or flexor (right) hot spot. Top, representative stick diagram decompositions of hindlimb movement for each condition; colors correspond to the period during which the modulated electrode was turned on. Diagram (left) shows calculation of hindlimb length, which combines changes over multiple joints. Bottom, electromyographic activity of extensor and flexor ankle muscles and the changes in hindlimb length for a series of steps, shown along with spatiotemporal pattern of stimulation; bar height is proportional to the stimulation amplitude. (b) Mean vertical ground reaction forces measured during stance with modulation of the amplitude of the extension electrode (top) and mean step height measured during swing with modulation of the flexion electrode (bottom). ${ }^{*} P<0.05$, one-way ANOVA; $n=5$, mean and s.e.m. (c) Vertical foot displacement during locomotion under different stimulation frequencies adjusted over a

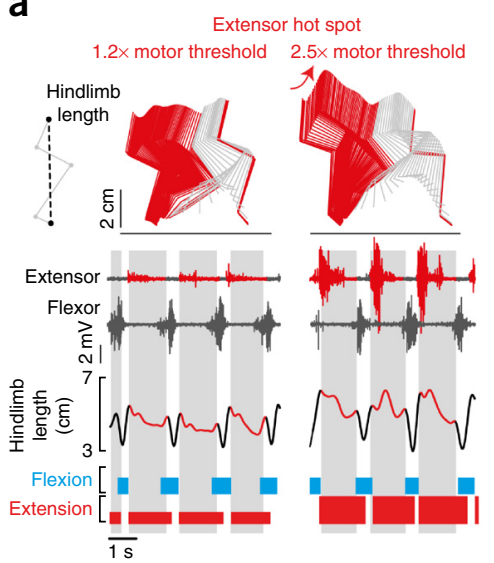

C

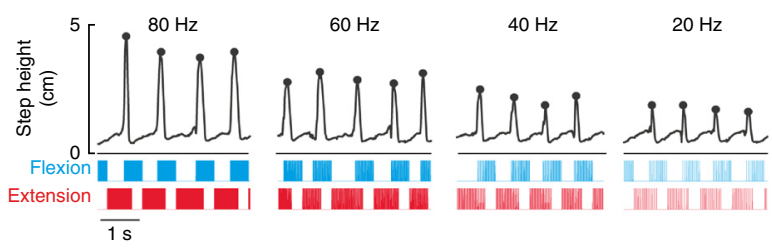

b
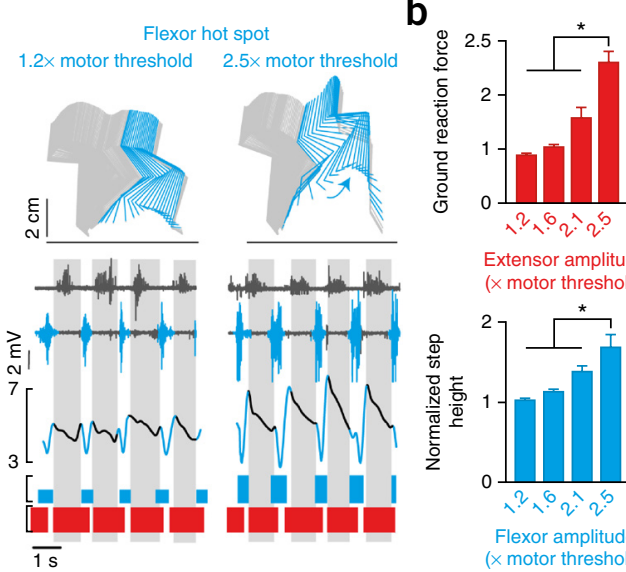

Extensor amplitude $(\times$ motor threshold $)$
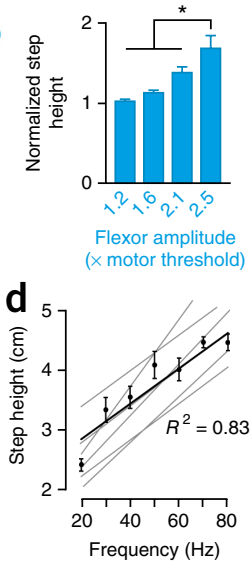

both extension and flexion electrodes. Dots highlight step height. Bars (bottom) show spatiotemporal patterns of stimulation. (d) Linear relationship between stimulation frequency and step height fit to the mean for all rats (black line) and individual rats (gray lines, $n=6$ ). 
Figure 6 Spatiotemporal neuromodulation improves motor control after clinically relevant $\mathrm{SCl}$. (a) Contusion in rat at T8. (b) Assessment of locomotion on a treadmill $14 \mathrm{~d}$ after injury. Continuous neuromodulation led to rapid fatigue, but spatiotemporal neuromodulation instantaneously restored locomotion.

(c) Histogram plots of maximum weight-bearing capacities, maximal vertical ground reaction forces and amplitude of extensor muscle activity measured in the same rats during continuous or spatiotemporal neuromodulation (mean and s.e.m.; $n=6,{ }^{*} P<0.05,{ }^{* *} P<0.01$, paired $t$-test). (d) Left, successive step heights measured during a sequence recorded in the same rat under both paradigms. Horizontal line and shaded areas, mean step height in intact rats $(n=3)$ and s.e.m. Right, bar plots of normalized duration of locomotion $(* * P<0.01$, paired $t$-test, $n=6$ ). (e) Trunk stability in rats as assessed through quadrupedal locomotion 2 months after $\mathrm{SCl}$. Parameters related to trunk dynamics were computed to obtain an index quantifying trunk stability relative to intact rats. The improvement of this index is shown for each rat individually $\left({ }^{* *} P<0.01\right.$, paired $t$-test, $n=6)$. (f) Stick diagram decomposition of hindlimb and trunk movements together with hindlimb endpoint trajectory during staircase climbing under spatiotemporal neuromodulation. Pie charts report the percentage of tumbles, touches and passes over the steps under both conditions. The percentage of successful passes over the step is shown for each rat individually (bottom right). ${ }^{* *} P<0.01$, paired $t$-test, $n=6$. a.u., arbitrary units. a
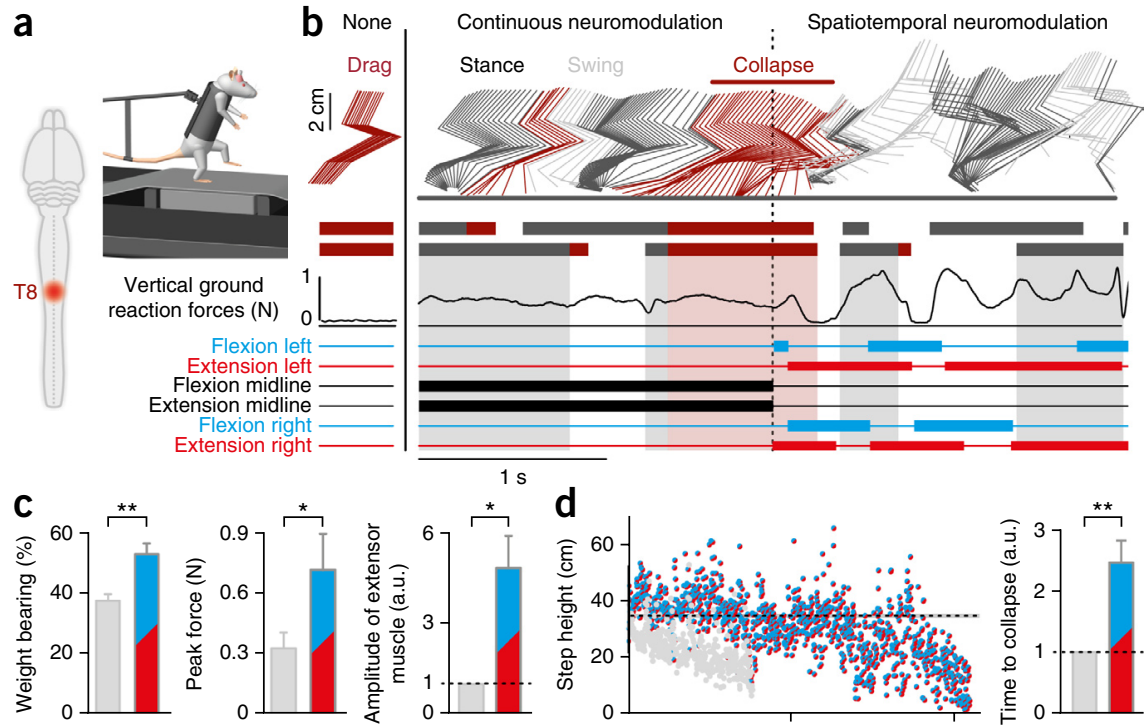

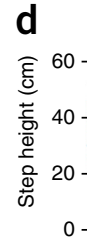
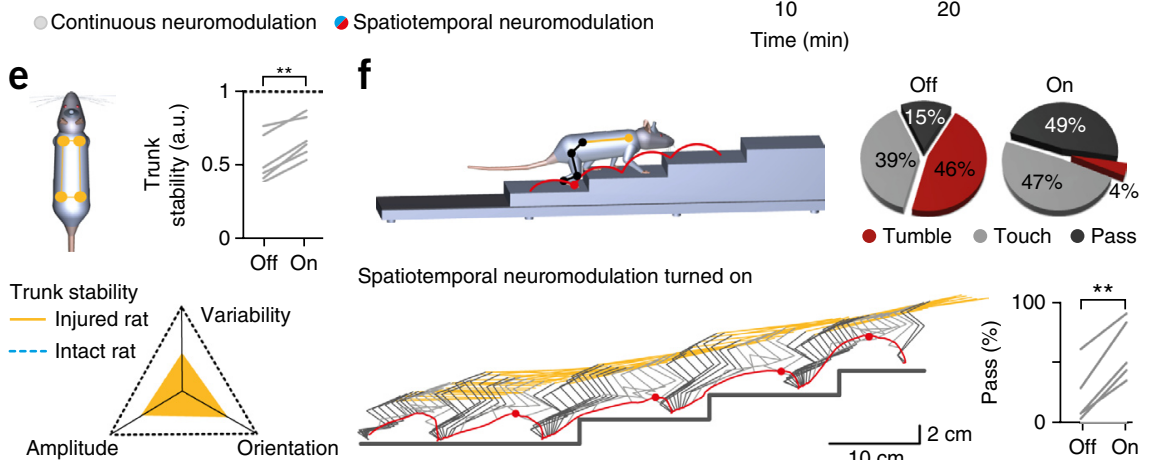

Spatiotemporal neuromodulation turned on

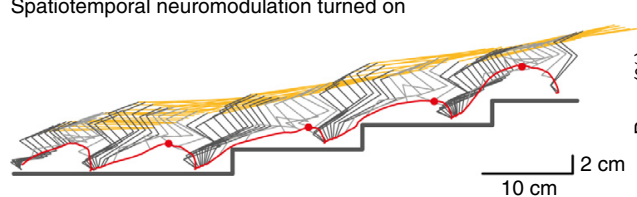

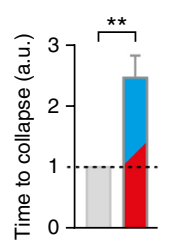

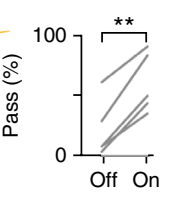

during spatiotemporal neuromodulation (Fig. 5c). In all rats, adjustment of stimulation frequency led to a gradual increase in both extension and flexion components, which mediated a linear tuning of gait features such as foot height (Fig. 5d). These relationships support control of complex foot trajectories during locomotion ${ }^{7}$ and can thus be combined with spatiotemporal neuromodulation.

\section{Spatiotemporal neuromodulation after clinically relevant $\mathrm{SCI}$}

Last, we evaluated the potential of spatiotemporal neuromodulation therapies to improve locomotion after clinically relevant SCI. Spinal cord damage in humans results primarily from contusions. To model such lesions, we delivered a robotically controlled impact to thoracic segments (21.8 \pm 1.7\% sparing) (Fig. 6a and Supplementary Fig. 10). Early after injury, all rats dragged both hindlimbs on the treadmill belt (Fig. 6b). Administration of serotoninergic replacement therapy did not promote movement at this stage. The addition of continuous electrical neuromodulation instantly enabled locomotion; however, hindlimb movements extinguished after a few minutes, as the rats were no longer able to support their body weight. Spatiotemporal neuromodulation instantly reestablished locomotion, enabling robust and powerful movements of both hindlimbs (Fig. $6 \mathbf{b}$ and Supplementary Video 3).

To quantify improvements in performance, we measured weightbearing capacities and endurance during continuous and spatiotemporal neuromodulation. To determine weight-bearing capacities, we progressively decreased the amount of vertical robotic assistance in $5 \%$ increments until the rats failed to perform ten consecutive steps. Spatiotemporal neuromodulation mediated 25-60\% augmentation of weight-bearing capacity across all rats tested $(P<0.05)$ (Fig. 6c), which correlated with substantial increase in vertical peak forces $(P<0.05)$ (Fig. 6c) and extensor muscle activity $(P<0.01)$ (Fig. 6c). To evaluate endurance, we provided optimal body weight support and measured the time until the rats collapsed. Spatiotemporal neuromodulation extended the duration of locomotion by $40-270 \%$ $(P<0.01)$ (Fig. 6d), maintaining step heights similar to those of intact rats during most of the testing (Fig. 6d).

In the chronic stage ( 8 weeks after injury), the rats showed limited recovery of hindlimb motor control. When tested quadrupedally on a runway, rats' gait performance ranged from limited movement of hindlimb joints to occasional plantar placements (Supplementary Fig. 11). During spatiotemporal neuromodulation, the rats were capable of full weigh-bearing plantar steps $(P<0.01)$ (Supplementary Fig. 11 and Supplementary Video 3). Spatiotemporal neuromodulation significantly improved trunk dynamics and thus the maintenance of balance during quadrupedal locomotion $(P<0.01)$ (Fig. 6e).

We then tested the rats during stair climbing, a task requiring precise voluntary foot placement over consecutive steps to resist gravity and avoid tumbling. Spatiotemporal neuromodulation allowed both hindlimbs to pass the steps successfully without contact with the staircase in a substantial number of trials $(P<0.01)$ (Fig. $6 \mathbf{f}$ and Supplementary Video 3).

\section{DISCUSSION}

Therapeutic outcomes in animal models $4,5,7,30,31$ and paraplegic patients $8,9,11,15$ have accelerated the deployment of clinical studies to evaluate the potential of epidural electrical stimulation for improving 
recovery after $\mathrm{SCI}^{32}$. In the absence of a conceptual framework, however, protocols have remained unspecific, with stimulation restricted to single regions and delivered continuously. Here, we have introduced spatiotemporal neuromodulation therapies derived from biological principles. This stimulation strategy reestablished lower-limb motor control where conventional protocols failed to facilitate movement. We expect this approach to support the design of evidence-based neuromodulation therapies that improve motor control and gait rehabilitation in humans.

Various studies have proposed that the motor command underlying the production of limb movement originates from a small set of motor primitives termed muscle synergies ${ }^{18,24,33,34}$. These motor primitives help cope with the redundant degrees of freedom at muscles and joints ${ }^{34}$ and are thus considered the building blocks of motor control ${ }^{24,33-35}$. Previous work suggests that a control architecture based on muscle synergies would simplify the manipulation of natural limb dynamics in robotics $^{26}$ and neuroprosthetics ${ }^{36}$. We followed this approach to design evidence-based neuromodulation therapies. As in humans ${ }^{36}$, we found that rodent locomotion involves the sequential activation of spatially restricted hot spots associated with extensor or flexor muscle synergies. These hot spots reflect the final motor command ${ }^{21}$, which is elaborated by neuronal circuits located in the same regions ${ }^{37-39}$. These cells integrate supraspinal information and feedback signals to produce precisely timed muscle contractions ${ }^{40-42}$. Evidence suggested that these cellular nodes encode muscle synergies promoting extension or flexion movements of the lower limbs ${ }^{37,38}$. These muscle synergy encoders receive dense synaptic contacts from proprioceptive afferents ${ }^{38,39}$. Indeed, the proprioceptive regulation of neuronally encoded motor primitives ${ }^{43}$ can predict the formation of lower-limb trajectory in the frog ${ }^{44}$.

Epidural electrical stimulation leads to motor pattern formation through the recruitment of proprioceptive feedback circuits, both in rats r,12,16,28 $^{\text {and humans }}{ }^{15,45}$. We reasoned that spinal implants designed to activate the proprioceptive afferents projecting to the hot spots identified would engage muscle synergy encoders related to extension and flexion. By analogy, the recruitment of auditory afferent pathways related to specific tones has guided the development of cochlear implants to restore hearing ${ }^{46}$. Tailored spinal implants with electrodes targeting specific subsets of dorsal roots enabled a gradual control over the degree of flexion and extension on the left and right hindlimbs. This hypothesis-driven neuromodulation therapy required only a few electrodes, whose locations were optimized through neuroanatomical reconstructions and computer simulations. In turn, the limited number of electrodes reduced technological challenges, allowing the development of highly reliable spinal implants that showed long-term functionality and bio-integration.

This conceptual framework also simplified the architecture of the real-time control software. We found that the temporal activation profiles of muscle synergies were captured in the spatiotemporal trajectory of the foot, as previously reported for human walking ${ }^{23}$. Thus, a single parameter provided a simplified and reliable feedback signal to trigger simultaneous adjustments of neuromodulation parameters across multiple electrode configurations with high fidelity.

These new concepts and technologies will support the development of more refined control policies. For example, we found that the predictive modulation of step height with changes in stimulation frequency ${ }^{7}$ was well preserved during spatiotemporal neuromodulation. Algorithms incorporating adjustments of spatial and temporal patterns, both in amplitude and frequency, under the guidance of dynamic computational models have the potential to further improve gait performance.
Habitual bipedalism elicited a series of adaptations in the dynamics of lower-limb motoneuron activation during human walking ${ }^{24,47}$. Despite these evolutionary adaptations, both muscle synergies ${ }^{24,35}$ and proprioceptive feedback circuits ${ }^{48}$ appear remarkably conserved across mammals. Moreover, the neuroanatomical ${ }^{49}$, electrophysiological ${ }^{15,17}$, computational ${ }^{18,24}$ and modeling ${ }^{13}$ concepts that guided the design of our hypothesis-driven neuromodulation therapies are directly applicable to humans.

Wearable and implanted wireless sensors ${ }^{50}$ provide off-the-shelf tools to monitor foot trajectory during locomotion. Neuromodulation platforms have been developed ${ }^{51}$ to enable integration of feedback signals for closed-loop electrical stimulation of the human nervous system. These neurotechnologies are well suited to clinical evaluations of the ability of spatiotemporal neuromodulation therapies to facilitate motor control and gait rehabilitation in humans. Although challenges lie ahead, we believe that spatiotemporal neuromodulation of the spinal cord will become a viable way to accelerate and augment functional recovery in humans with SCI.

\section{METHODS}

Methods and any associated references are available in the online version of the paper.

Note: Any Supplementary Information and Source Data files are available in the online version of the paper.

\section{ACKNOWLEDGMENTS}

We thank people who have helped over the past 6 years to take care of injured animals and to test multiple iterations of neural implants. We also would like to thank D. Pioletti for providing access to the microcomputed tomography scanner. N.W. is a participant in the Charité Clinical Scientist Program funded by the Charité-Universitätsmedizin Berlin and the Berlin Institute of Health. Funding was provided by the European Commission's Seventh Framework Programme (CP-IP 258654, NeuWALK, G.C., S.M., E.B., J.B. and P.D.); a Starting Grant from the European Research Council (ERC 261247, Walk Again, G.C.); the Russian Science Foundation (RSF grant 14-15-00788, P.M.) and the Swiss National Science Foundation Centre of Competence in Research (NCCR) in Robotics (G.C., S.L., S.M.), Dynamo project (S.M.) and NanoTera.ch program (SpineRepair) (G.C., S.L. and S.M.).

\section{AUTHOR CONTRIBUTIONS}

P.M. and M.C. contributed equally as second authors to this work. N.W., P.M., J.B., N.P. and G.C. developed and performed the surgeries. N.W., E.M.M., J.G., P.M., J.D., L.B., S.D., M.C., L.A. and G.C. performed in vivo experiments. N.W., E.M.M., J.G., C.G.L.G., L.A., N.D. and G.C. analyzed functional data. N.W., E.M.M. and J.G. conducted the statistical analysis. S.P.L., P.D., I.R.M., A.H., P.M., O.H., S.K. and F.S. developed the implant technology. M.C., A.M. and S.M. conducted computational simulations. J.K., Q.B. and N.P. conducted anatomical evaluations. N.W., E.M.M., P.M., M.C., J.D., S.M., E.B. and G.C. conceived experiments. R.v.d.B., E.B., J.B., L.B. and S.D. were responsible for the animal models. N.W., E.M.M., L.A., J.G., C.G.L.G., and G.C. prepared the figures with the help of the other authors. G.C. wrote the manuscript, and all authors contributed to its editing. G.C. supervised and coordinated all the aspects of the work.

\section{COMPETING FINANCIAL INTERESTS}

The authors declare competing financial interests: details are available in the online version of the paper.

Reprints and permissions information is available online at http://www.nature.com/ reprints/index.html.

1. Borton, D., Micera, S., Millán, Jdel.R. \& Courtine, G. Personalized neuroprosthetics Sci. Transl. Med. 5, 210 rv2 (2013).

2. Lozano, A.M. \& Lipsman, N. Probing and regulating dysfunctional circuits using deep brain stimulation. Neuron 77, 406-424 (2013).

3. Barthélemy, D., Leblond, H. \& Rossignol, S. Characteristics and mechanisms of locomotion induced by intraspinal microstimulation and dorsal root stimulation in spinal cats. J. Neurophysiol. 97, 1986-2000 (2007).

4. Courtine, G. et al. Transformation of nonfunctional spinal circuits into functional states after the loss of brain input. Nat. Neurosci. 12, 1333-1342 (2009). 
5. van den Brand, R. et al. Restoring voluntary control of locomotion after paralyzing spinal cord injury. Science 336, 1182-1185 (2012).

6. Holinski, B.J., Everaert, D.G., Mushahwar, V.K. \& Stein, R.B. Real-time control of walking using recordings from dorsal root ganglia. J. Neural Eng. 10, 056008 (2013).

7. Wenger, N. et al. Closed-loop neuromodulation of spinal sensorimotor circuits controls refined locomotion after complete spinal cord injury. Sci. Transl. Med. 6, 255 ra133 (2014).

8. Carhart, M.R., He, J., Herman, R., D'Luzansky, S. \& Willis, W.T. Epidural spinal-cord stimulation facilitates recovery of functional walking following incomplete spinalcord injury. IEEE Trans. Neural Syst. Rehabil. Eng. 12, 32-42 (2004).

9. Angeli, C.A., Edgerton, V.R., Gerasimenko, Y.P. \& Harkema, S.J. Altering spinal cord excitability enables voluntary movements after chronic complete paralysis in humans. Brain 137, 1394-1409 (2014).

10. Gerasimenko, Y.P. et al. Noninvasive reactivation of motor descending control after paralysis. J. Neurotrauma 32, 1968-1980 (2015).

11. Herman, R., He, J., D'Luzansky, S., Willis, W. \& Dilli, S. Spinal cord stimulation facilitates functional walking in a chronic, incomplete spinal cord injured. Spinal Cord 40, 65-68 (2002).

12. Capogrosso, M. et al. A computational model for epidural electrical stimulation of spinal sensorimotor circuits. J. Neurosci. 33, 19326-19340 (2013).

13. Rattay, F., Minassian, K. \& Dimitrijevic, M.R. Epidural electrical stimulation of posterior structures of the human lumbosacral cord: 2. Quantitative analysis by computer modeling. Spinal Cord 38, 473-489 (2000).

14. Ladenbauer, J., Minassian, K., Hofstoetter, U.S., Dimitrijevic, M.R. \& Rattay, F. Stimulation of the human lumbar spinal cord with implanted and surface electrodes: a computer simulation study. IEEE Trans. Neural Syst. Rehabil. Eng. 18, 637-645 (2010).

15. Hofstoetter, U.S. et al. Periodic modulation of repetitively elicited monosynaptic reflexes of the human lumbosacral spinal cord. J. Neurophysiol. 114, 400-410 (2015).

16. Gerasimenko, Y.P. et al. Spinal cord reflexes induced by epidural spinal cord stimulation in normal awake rats. J. Neurosci. Methods 157, 253-263 (2006).

17. Sayenko, D.G., Angeli, C.A., Harkema, S.J., Edgerton, V.R. \& Gerasimenko, Y.P. Neuromodulation of evoked muscle potentials induced by epidural spinal cord stimulation in paralyzed individuals. J. Neurophysiol. 111, 1088-1099 (2014).

18. Danner, S.M. et al. Human spinal locomotor control is based on flexibly organized burst generators. Brain 138, 577-588 (2015).

19. Edgerton, V.R. et al. Training locomotor networks. Brain Res. Rev. 57, 241-254 (2008).

20. Rejc, E., Angeli, C. \& Harkema, S. Effects of lumbosacral spinal cord epidural stimulation for standing after chronic complete paralysis in humans. PLOS ONE 10, e0133998 (2015).

21. Yakovenko, S., Mushahwar, V., VanderHorst, V., Holstege, G. \& Prochazka, A. Spatiotemporal activation of lumbosacral motoneurons in the locomotor step cycle. J. Neurophysiol. 87, 1542-1553 (2002).

22. Cappellini, G., Ivanenko, Y.P., Dominici, N., Poppele, R.E. \& Lacquaniti, F. Migration of motor pool activity in the spinal cord reflects body mechanics in human locomotion. J. Neurophysiol. 104, 3064-3073 (2010).

23. Ivanenko, Y.P. et al. Temporal components of the motor patterns expressed by the human spinal cord reflect foot kinematics. J. Neurophysiol. 90, 3555-3565 (2003).

24. Dominici, N. et al. Locomotor primitives in newborn babies and their development. Science 334, 997-999 (2011).

25. Kiehn, O. Locomotor circuits in the mammalian spinal cord. Annu. Rev. Neurosci. 29, 279-306 (2006)

26. Berniker, M., Jarc, A., Bizzi, E. \& Tresch, M.C. Simplified and effective motor control based on muscle synergies to exploit musculoskeletal dynamics. Proc. Natl. Acad. Sci. USA 106, 7601-7606 (2009).

27. Lee, D.D. \& Seung, H.S. Learning the parts of objects by non-negative matrix factorization. Nature 401, 788-791 (1999).

28. Lavrov, I. et al. Epidural stimulation induced modulation of spinal locomotor networks in adult spinal rats. J. Neurosci. 28, 6022-6029 (2008).
29. Minassian, K. et al. Stepping-like movements in humans with complete spinal cord injury induced by epidural stimulation of the lumbar cord: electromyographic study of compound muscle action potentials. Spinal Cord 42, 401-416 (2004).

30. Ichiyama, R.M. et al. Step training reinforces specific spinal locomotor circuitry in adult spinal rats. J. Neurosci. 28, 7370-7375 (2008).

31. Gad, P. et al. Forelimb EMG-based trigger to control an electronic spinal bridge to enable hindlimb stepping after a complete spinal cord lesion in rats. J. Neuroeng. Rehabil. 9, 38 (2012).

32. Consortium for Addressing Paralysis through Spinal Stimulation Technologies. Framework for a Research Study on Epidural Spinal Stimulation to Improve Bladder, Bowel, and Sexual Function in Individuals with Spinal Cord Injuries. (National Institutes of Health, 2015).

33. Bizzi, E., Tresch, M.C., Saltiel, P. \& d'Avella, A. New perspectives on spinal motor systems. Nat. Rev. Neurosci. 1, 101-108 (2000).

34. Bernstein, N. The Co-ordination and Regulation of Movements (Pergamon, 1967).

35. Giszter, S.F. Motor primitives - new data and future questions. Curr. Opin. Neurobiol. 33, 156-165 (2015).

36. La Scaleia, V., Ivanenko, Y.P., Zelik, K.E. \& Lacquaniti, F. Spinal motor outputs during step-to-step transitions of diverse human gaits. Front. Hum. Neurosci. 8 305 (2014).

37. Hägglund, $M$. et al. Optogenetic dissection reveals multiple rhythmogenic modules underlying locomotion. Proc. Natl. Acad. Sci. USA 110, 11589-11594 (2013).

38. Levine, A.J. et al. Identification of a cellular node for motor control pathways. Nat. Neurosci. 17, 586-593 (2014).

39. Tripodi, M., Stepien, A.E. \& Arber, S. Motor antagonism exposed by spatial segregation and timing of neurogenesis. Nature 479, 61-66 (2011).

40. Grillner, S. \& Jessell, T.M. Measured motion: searching for simplicity in spinal locomotor networks. Curr. Opin. Neurobiol. 19, 572-586 (2009).

41. Arber, S. Motor circuits in action: specification, connectivity, and function. Neuron 74, 975-989 (2012).

42. Bourane, S. et al. Identification of a spinal circuit for light touch and fine motor control. Cell 160, 503-515 (2015).

43. Hart, C.B. \& Giszter, S.F. A neural basis for motor primitives in the spinal cord. J. Neurosci. 30, 1322-1336 (2010).

44. Kargo, W.J., Ramakrishnan, A., Hart, C.B., Rome, L.C. \& Giszter, S.F. A simple experimentally based model using proprioceptive regulation of motor primitives captures adjusted trajectory formation in spinal frogs. J. Neurophysiol. 103, 573-590 (2010).

45. Sayenko, D.G., Angeli, C., Harkema, S.J., Edgerton, V.R. \& Gerasimenko, Y.P. Neuromodulation of evoked muscle potentials induced by epidural spinal-cord stimulation in paralyzed individuals. J. Neurophysiol. 111, 1088-1099 (2014).

46. Zeng, F.G., Rebscher, S., Harrison, W., Sun, X. \& Feng, H. Cochlear implants: system design, integration, and evaluation. IEEE Rev. Biomed. Eng. 1, 115-142 (2008).

47. Capaday, C. The special nature of human walking and its neural control. Trends Neurosci. 25, 370-376 (2002).

48. Clarac, F., Cattaert, D. \& Le Ray, D. Central control components of a 'simple' stretch reflex. Trends Neurosci. 23, 199-208 (2000).

49. Canbay, S. et al. Anatomical relationship and positions of the lumbar and sacral segments of the spinal cord according to the vertebral bodies and the spinal roots. Clin. Anat. 27, 227-233 (2014).

50. Mariani, B., Jiménez, M.C., Vingerhoets, F.J. \& Aminian, K. On-shoe wearable sensors for gait and turning assessment of patients with Parkinson's disease. IEEE Trans. Biomed. Eng. 60, 155-158 (2013).

51. Afshar, P. et al. A translational platform for prototyping closed-loop neuromodulation systems. Front. Neural Circuits 6, 117 (2012). 


\section{ONLINE METHODS}

Animal models and surgical procedures. Animal species and animal groups. All experiments were conducted in accordance with Swiss federal legislation and under the guidelines established at EPFL. Local Swiss Veterinary Offices approved all the procedures. Experiments were performed on female Lewis rats (LEW-ORlj) with initial weight of 180-220 g (Janvier Labs). A total of 47 rats were used for experiments, distributed as follows: experiments to determine the spatial distribution of hindlimb motoneurons, $n=20$ rats; experiments for reconstruction of spinal segments and dorsal roots $n=3$ rats; experiments for motor-evoked potentials and induction of movement, $n=6$ rats; experiments to determine the spatiotemporal map of motoneuron activation in healthy animals, $n=7$ rats; experiments to test the functional effects of spatiotemporal neuromodulation in rats with complete SCI, $n=5$ rats, contusion SCI, $n=6$ rats.

Surgical procedures. General surgical procedures for the majority of interventions carried out have been described previously ${ }^{5,7,52}$. All interventions were performed under full general anesthesia with isoflurane in oxygen-enriched air (1-2\%). After surgery, the rats were placed in an incubator for optimized recovery from anesthesia.

Implantation of intramuscular electrodes. To record electromyographic activity, bipolar electrodes were implanted into hindlimb muscles. The following muscles were implanted in different combinations depending on experiments: gluteus medius, iliopsoas, vastus lateralis, semitendinosus, biceps femoris, gastrocnemius medialis, gastrocnemius lateralis, tibialis anterior, extensor digitorum longus and flexor hallucis longus. Briefly, recording electrodes were fabricated by removing a small part (0.5-mm notch) of insulation from each the implanted wire (AS631, Cooner Wire). A common ground wire was created by removing about $1 \mathrm{~cm}$ of Teflon from the distal extremity of the wire, which was inserted subcutaneously over the right shoulder. All electrode wires were connected to a percutaneous amphenol connector (Omnetics Connector Corporation) cemented to the skull of the rats. The proper location of the electrodes was verified postmortem.

Surgical insertion of spinal implants. The surgical methods to insert and stabilize the spinal implant into the epidural space are detailed in Supplementary Figure 6. Briefly, two partial laminectomies were performed at vertebrae levels L3-L4 and T12-T13 to create entry and exit points for the implant. The implant was gently pulled above the dura mater using a surgical suture. Electrophysiological testing was performed intra-operatively to fine-tune positioning of electrodes. The connector of the implant was secured into a protective cage plastered using freshly mixed dental cement on top of the L3-L4 vertebra.

Spinal cord injury models. Two models of spinal cord injuries were used in the present experiments. All the main experiments were conducted in rats that received a complete transection of the mid-thoracic spinal cord, toward T8 spinal segment. Under aseptic conditions and general anesthesia, a dorsal midline skin incision was made from vertebral level T5 to L2, and the underlying muscles were removed. A partial laminectomy was performed around T8 vertebra to expose the spinal cord. The exposed spinal cord was then cut with customized tools. The complete transection lesions were verified postmortem by confirming the absence of neural tissues throughout the dorsoventral extent of the spinal cord. The second group of rats received a spinal cord contusion. A partial laminectomy was made at the T9 vertebra to expose T7 andT8 segments. A 230 kdyn $(1$ dyn $=10 \mu \mathrm{N})$ contusion injury was applied using a force-controlled spinal cord impactor (IH-0400 Impactor, Precision Systems and Instrumentation LLC, USA). The amount of damaged spinal tissue was evaluated using coronal sections stained with antibodies against glial fibrillary acidic protein (GFAP) (Dako Z0334, USA). The contours of the spared and damaged spinal tissues were reconstructed in Neurolucida image analysis software to reconstruct the lesion in 3D.

Rehabilitation procedures after spinal cord injury. All the injured rats were trained daily for $30 \mathrm{~min}$, starting $7 \mathrm{~d}$ after injury. The neurorehabilitation program was conducted on a treadmill using a robotic body weight support system (Robomedica) that was adjusted to provide optimal assistance during bipedal stepping ${ }^{7}$. To enable locomotion, a serotonergic replacement therapy combining the $5 \mathrm{HT}_{2 \mathrm{~A}}$ agonist quipazine and the $5 \mathrm{HT}_{1 \mathrm{~A}-7}$ agonist 8 -OHDPAT was administered systemically $10 \mathrm{~min}$ before training. Electrical neuromodulation was delivered throughout the training session.
Multimodal recordings and data analysis. Recordings of whole-body kinematics, kinetics and muscle activity. Procedures to record kinematics, kinetics and muscle activity have been detailed previously ${ }^{7}$. Briefly, whole-body kinematics was recorded using the high-speed motion capture system Vicon (Vicon Motion Systems), combining 12 infrared cameras $(200 \mathrm{~Hz})$. Reflective markers were attached bilaterally overlying the iliac crest, greater trochanter (hip), lateral condyle (knee), lateral malleolus (ankle) and distal end of the fifth metatarsal (limb endpoint). During quadrupedal locomotion, an additional marker was placed on the shoulders. 3D position of the markers was reconstructed offline using Vicon Nexus software. The body was modeled as an interconnected chain of rigid segments, and joint angles were generated accordingly. In addition, we computed changes in the angle and length of the virtual telescopic limb linking the hip to the foot (hindlimb). Electromyographic signals $(2 \mathrm{kHz})$ were amplified, filtered (10-2,000 Hz bandpass), stored and analyzed offline. Ground reaction forces in the vertical, anteroposterior and mediolateral directions were monitored using a force plate ( $2 \mathrm{kHz}$, HE6X6, AMTI) located in the middle of the runway or below the treadmill belt. Concurrent video recordings $(200 \mathrm{~Hz})$ were obtained using two cameras (Basler Vision Technologies) oriented at 90 degrees and 270 degrees with respect to the direction of locomotion.

Analysis of whole-body kinematics, kinetics and muscle activity. A minimum of 10 step cycles was typically extracted for each hindlimb in each experimental task and rat. A total of 137 parameters quantifying kinematics $(n=118)$ and kinetic $(n=6)$ and muscle activity $(n=13)$ features were computed for each gait cycle using custom-written Matlab scripts and according to methods described previously $^{7}$ (all the computed parameters are reported in Supplementary Table 1). To demonstrate the effects of experimental conditions and extract the parameters that captured the largest amount of variance across rats or between conditions, we implemented a multistep procedure based on principal component (PC) analysis ${ }^{5,7,53}$. PC analyses were applied on all the computed parameters from all individual gait cycles for all the rats together. Gait cycles were visualized in the new synthetic PC space, and PC scores were extracted to quantify differences between groups or experimental conditions. Parameters highly correlated (factor loadings) with PCs that discriminated the experimental conditions were extracted and regrouped into functional clusters corresponding to basic movement features.

Dynamic trunk balance. To quantify improvement of dynamic trunk movement during quadrupedal locomotion, we computed the mean of an ensemble of parameters related to the orientation of the trunk, the variability of trunk movement and the amplitude of trunk movement. All parameters are reported in Supplementary Table 1. For each component of trunk movement (amplitude, orientation and variability), we expressed these parameters along a dedicated axis that reports the degree of similarity of these parameters to those measured in intact rats (Fig. 6e).

Muscle synergy extraction. Muscle activation signals (electromyographic data) were high-pass filtered $(30 \mathrm{~Hz})$ with a zero-lag fourth-order Butterworth filter, full-wave rectified, smoothed with a zero-lag fourth-order low-pass $(20 \mathrm{~Hz})$ Butterworth filter, time interpolated over individual gait cycles to fit a normalized time base with 200 points and averaged across all gait cycles performed by the individual rat. The electromyographic signal of each muscle was normalized to its peak value. We then applied a non-negative matrix factorization algorithm on these curated muscle activation signals to derive the weighting components and temporal activation profiles of muscle synergies ${ }^{24}$.

Spatiotemporal map of motoneuron activation. To visualize spatiotemporal map of motoneuron activation, electromyographic signals were mapped onto the rostrocaudal distribution of the reconstructed motoneuron matrix ${ }^{21}$. This approach provides an interpretation of the motoneuron activation at a segmental level rather than at the individual muscle level. The maps were constructed by adding the contributions of each muscle to the total activity at each spinal segment. The motor output pattern of each spinal segment Si was estimated by the following equation:

$$
S_{i}=\frac{\sum_{j=1}^{n i}\left(\frac{M N i j}{M N j}\right) \cdot E M G j}{\sum_{j=1}^{n i}\left(\frac{M N i j}{M N j}\right)} \cdot N i
$$


where ni is the number of EMGjs corresponding to the $i$ th segment, EMGj represents the normalized muscle activity, MNij is the number of motor neurons related to muscle $j$ for the segment $i, \mathrm{MNj}$ is the total number of motor neurons for the muscle $j, \mathrm{Ni}$ is the number of muscles innervated by the $i$ th spinal segment.

Gaussian cluster algorithms. Clusters of activity were extracted from the spatiotemporal map of motoneuron activation using Gaussian mixture models (GMMs). GMMs were applied to the temporal activation profiles of motoneuron activation within each spinal segment. Four clusters maximized fitting quality according to Bayesian information criteria. This analysis highlighted two main hot spots along the rostrocaudal direction, and three main timings of activation corresponding to stance, stance-to-swing transition, and late swing.

Anatomical and imaging procedures. Spatial distribution of hindlimb motoneurons. Rats $(n=20)$ received injections of Fluorogold ( $2 \%$ in sterile saline, $30-80 \mu \mathrm{l}$ per muscle) into the tibialis anterior muscle, and in the same surgery, into one additional muscle on the contralateral side. The following muscles were traced: gluteus medius, iliopsoas, vastus lateralis, semitendinosus, biceps femoris, gastrocnemius medialis, gastrocnemius lateralis, extensor digitorum longus, and flexor hallucis longus. The location of the retrogradely traced motoneurons was reconstructed in $3 \mathrm{D}$ from serial spinal cord sections using Neurolucida. To merge reconstruction from several rats into a unified digital library, the tibialis anterior motor column was used as a landmark in each rat.

Microcomputed tomography. Repeated imaging of spinal implants in vivo was conducted using the microcomputed tomography scanner Skyscan 1076 (Bruker $\mu \mathrm{CT}$ ). Scanner settings were adjusted to avoid artifacts induced by metallic parts of the vertebral orthosis $(0.5-1 \mathrm{~mm}$ aluminum filter, voltage $70-100 \mathrm{kV}$, current $100-140 \mu \mathrm{A}$, exposure time $120-160 \mathrm{~ms}$, rotation step $\left.0.5^{\circ}\right)$. The resulting projection images were reconstructed into $3 \mathrm{D}$ renderings using NRecon and GPURecon Server (Bruker $\mu \mathrm{CT}$ ). Rats were kept under isoflurane anesthesia during the scan to reduce motion artifacts. Segmentation and 3D model were constructed with Amira (FEI Vizualisation Sciences Group).

Measurement of vertebrae and spinal segments. The shape of vertebrae was measured using microcomputed tomography imaging. The spinal cords of three rats were imaged, and the entire bone structure was reconstructed in $3 \mathrm{D}$. The $3 \mathrm{D}$ renderings were exported in the virtual reality modeling language file format WRL that was later merged with spinal tissue and dorsal root reconstructions.

Measurements of relationships between vertebra and spinal segment morphologies were performed on fresh tissue. For each subject, the spinal segments were identified on the basis of the innervation of the dorsal roots. The center of the segment was defined as the entry point of the rootlets. After measuring the length of vertebra and the relationships between vertebra and spinal segments, the entire spinal cord was extracted and the roots moved perpendicular to the spinal cord to clearly visualize the segments. The location and length of each segment was then calculated.

Reconstruction of spinal segments. At the end of the experimental procedures, rats were perfused with Ringer's solution containing 100,000 IU/L heparin and $0.25 \% \mathrm{NaNO}_{2}$ followed by $4 \%$ phosphate-buffered paraformaldehyde, $\mathrm{pH} 7.4$, containing $5 \%$ sucrose. The spinal cords were dissected, postfixed overnight and transferred to $30 \%$ phosphate buffered sucrose for cryoprotection. After $4 \mathrm{~d}$, the tissue was embedded and the entire lumbosacral spinal cord sectioned in a cryostat at a $40-\mu \mathrm{m}$ thickness. To reconstruct spinal segment morphology in a 3D model, a Nissl staining (ThermoFisher) was performed on 25 even crosssections of lumbar and sacral segments, each separated by $0.8 \mathrm{~mm}$. The slides were assembled into the Neurolucida image analysis software (MBF Bioscience) to reconstruct lumbosacral segments in 3D.

Reconstruction of dorsal root trajectories. After fixation, the spinal cord was extracted from the vertebral canal. The dura mater was opened along the rostrocaudal axis and gently moved on the side. For each spinal segment, the dorsal root ganglia were identified. The corresponding root was retracted cranially and laterally. The entire length of the root was painted, from the cut extremity to the entrance into the spinal segment. All the painted roots from S3 to L1 were repositioned in their original location along spinal segments, and the dura mater was sutured using surgical wire (Ethilon 6.0). The spinal cord was then frozen and cut into $80-\mu \mathrm{m}$ thick slices using a cryostat (Leica Instruments). Reconstructions were conducted using every other section, corresponding to intervals of $160 \mu \mathrm{m}$.
The slides were assembled into the Neurolucida image analysis software (MBF Bioscience) to reconstruct the color-coded dorsal roots in 3D.

Histology of explanted spinal cord with long-term implants. After 8 weeks of implantation, rats were perfused and their spinal cords sectioned as described above. Astrocytic reactivity was revealed by immunohistological staining against glial fibrillary acidic protein (GFAP). Coronal sections of the spinal segments located below the implant were incubated overnight in serum containing anti-GFAP (1:1,000, Dako Z0334) antibodies. Immunoreactions were visualized with appropriate secondary antibodies labeled with Alexa Fluor 488 or Alexa Fluor 555 (Thermo Fisher). Sections were mounted onto microscope slides using anti-fade fluorescent mounting medium and covered with a cover glass. The tissue sections were observed and photographed with a laser confocal fluorescence microscope (Leica). Immunostaining density was measured offline using 6 representative confocal images of each lumbar segment per rat. Images were acquired using standard imaging settings that were kept constant across rats and analyzed using custom-written Matlab scripts according to previously described methods 5 . All analyses were performed blindly.

Morphology of the entire spinal cord. All the 3D reconstructions derived from microcomputed tomography imagining and anatomical experiments were merged to generate a global model including the bony structure of vertebrae, the shape of spinal segments, and the trajectory of each dorsal root. We generated a database using the STL file format, which is supported by many software packages and is widely used for rapid prototyping and computer-aided manufacturing. This database is available for download at http://courtine-lab.epfl.ch.

Computational model. Hybrid computational model of electrical spinal cord stimulation. We previously elaborated and validated experimentally a hybrid computational model of epidural electrical stimulation of the rat spinal cord ${ }^{12}$ The model combines (i) finite element modeling of the entire lumbosacral spinal cord to compute the electrical fields elicited by electrical spinal cord stimulation, and (ii) anatomically and biophysically realistic neural structures to derive the type of fibers and neurons activated by the stimulation. In the present experiments, we integrated all the results from the traced hindlimb motoneurons (Supplementary Fig. 1) and updated the positioning of the dorsal roots in the model with the new $3 \mathrm{D}$ reconstructions.

Computer simulations and model predictions. We conducted simulations to identify the optimal electrode locations to activate interneurons and motoneurons located within extensor- and flexor-related hot spots of lumbar segments during gait. Computerized simulations, neurophysiological experiments and pharmacological testing revealed that epidural electrical stimulation activates interneurons and motoneurons indirectly through the recruitment of proprioceptive fibers feedback circuits fibers within the dorsal roots ${ }^{7,12,28}$. Therefore, we performed computerized simulations that searched optimal electrode locations to activate proprioceptive afferent fibers (A $\alpha$ fibers) running in the dorsal roots projecting to the targeted spinal segments. The segmental distribution of extensor- and flexor-related hot spots during gait was derived from Gaussian clustering analysis (Fig. 1).

To optimize electrode configurations, a grid of electrodes was positioned over the modeled lumbosacral segments. The grid combined five active sites per segment, resulting in a total of 30 electrodes. Active sites were located over the midline, and at 750 and $1,500 \mu \mathrm{m}$ from the midline for the more caudal segments (L4-S1), and at 1,000 and $1,500 \mu \mathrm{m}$ from the midline for the more rostral segments (L2-L3) in order to conform the enlargement of lumbosacral segments. The spacing of electrodes along the mediolateral direction was selected to maximize left-right specificity ${ }^{12}$. To evaluate the ability of each electrode to access the targeted dorsal roots, we elaborated an optimization algorithm that calculated the cost to achieve specificity on the basis of four factors: (i) stimulation specificity for the targeted segments, (ii) ipsilateral versus contralateral specificity, (iii) minimum threshold and (iv) specificity of the neighboring sites. The last term was important to ensure robustness in case of electrode misplacement, inter-individual variability and post-implantation migration. For each active site, stimulations were delivered at increasing amplitudes ranging from 50 to $600 \mu \mathrm{A}$. The resulting recruitment of Ia- $\alpha$ fibers was evaluated for each segment at each current level. The total number of implemented simulations reached 112,500 , which required approximately $8 \mathrm{~d}$ of computer time (Supplementary Fig. 4). 
Acute electrophysiological experiments. Motor-evoked potentials to validate model predictions. Adult rats were anesthetized with urethane $(1 \mathrm{~g} / \mathrm{kg}$, i.p., SigmaAldrich). Bipolar electrodes were implanted into a pair of antagonist muscles spanning each joint of the hindlimb: gluteus medius, iliopsoas, vastus lateralis, semitendinosus, gastrocnemius medialis, tibialis anterior, extensor digitorum longus and flexor hallucis longus. An extensive laminectomy was performed to expose all the lumbar and sacral segments and visualize the dorsal roots projecting to each segment. Motor-evoked potentials were simultaneously recorded in all the implanted muscles while delivering rectangular pulses (0.2-ms duration) at $0.5 \mathrm{~Hz}$ through epidural electrodes. The electrode was positioned at the locations identified by simulations using dorsal roots and the spinal cord midline as anatomical landmarks. The intensity of the electrical stimulation was increased progressively from 20 to $300 \mu \mathrm{A}$. Electromyographic signals $(12.207 \mathrm{kHz})$ were amplified, filtered (1-5,000 $\mathrm{Hz}$ bandpass), and stored for offline analysis. The root mean square (RMS) of the motor-evoked potentials were calculated for each muscle, and represented in color-coded spatial maps of motoneuron activation that were directly meshed onto the spinal cord model. The methodology to compute these maps is explained above.

Spinal implants. Epidural implants were fabricated using ultraviolet (UV) photolithographic patterning of a photosensitive polyimide, as well as microelectroforming to create gold electrodes and embedded gold interconnects. Polyimide is a mechanically and chemically robust polymer material that showed a high level of biocompatibility ${ }^{54}$. Processing of the implants was performed on wafer scale, which allowed parallel fabrication of ten devices or more on a $125-\mathrm{mm}$ silicon wafer serving as carrier wafer. Processing started with deposition of a $20-\mu \mathrm{m}$ thick polyimide film by spin coating on the silicon substrate. UV lithography was used to shape the polyimide-based bottom layer, which constituted the footprint of the implant. A 200 -nm thick gold layer was deposited using vacuum evaporation and lithographically structured providing a conductive seed pattern. Gold was then electroplated to a height of approximately $6 \mu \mathrm{m}$ to create the electrodes and leads. A $20-\mu \mathrm{m}$ cover layer of photosensitive polyimide resist was applied by spin coating to uniformly cover the bottom and electrode structure. A final UV lithography step was conducted to structure the top layer of the implant, and create openings over the electrodes and contact pads. Before peeling off the implants from the carrier wafer, holes were punctuated through the polyimide using mechanical punching to promote the growth of conjunctive tissues that stabilized implants over time. To create a connector, the contact pads located at the extremity of the implant were glued to a small support plate made of medical-grade PMMA sheet material. Precision-milled alignment structures allowed fine adjustment of the support plate to the polyimide-based implant, and to the extremity of the leads. The support plate contained a seat in which a tiny printed circuit board (PCB) with conductive stripes was fixed. Stainless steel leads with FEP (fluorinated ethylene propylene) insulation (AS-632, Cooner Wire) were directly soldered to the contact pads of the implant via the contact stripes located on the PCB. The resulting implants and connective leads were highly reliable mechanically and electrically. Finally, the entire contact area was covered with a UV-curable, methacrylate-based resin that provided tight sealing. For improved biointegration, the contact interface was over-molded with a thin layer of medical-grade silicone (Supplementary Fig. 4).

Long-term functionality of spinal implants. Induction of movement and motorevoked potentials. The ability of spinal implants to engage specific muscles and to elicit distinct movements was tested using short bursts of stimulation delivered through chronic spinal implants. After recovery from implantation ( 2 weeks), rats $(n=6)$ were lightly anesthetized with ketamine and suspended in the air in a custom-made jacket. Electromyographic activity was recorded at $2 \mathrm{kHz}$ in conjunction with bilateral hindlimb kinematics (described above). Stimulations were delivered at $40 \mathrm{~Hz}$ and synchronized with recordings of motor-evoked potentials and kinematic data. Stimulation conditions were randomized and blinded to the experimenter. To avoid repeated anesthesia of animals, experiments were repeated 4 times on one day.

To verify the long-term functionality of electrodes, motor-evoked potentials were recorded weekly in fully awake rats. For each electrode of the spinal implant, single pulses $(0.2 \mathrm{~ms}, 0.5 \mathrm{~Hz})$ were delivered at increasing intensities. The current threshold necessary to elicit motor-evoked potentials in the left and right tibialis anterior muscles was measured. The values obtained were normalized to baseline recordings and averaged across rats and electrodes.

Electrode impedance. The impedance of electrodes embedded in the spinal implants was recorded using a bipolar electrode configuration. The counter electrode was created by removal of the insulation of a Teflon-coated stainless wire at its extremity and implanted the electrode subcutaneously. Impedance at $1 \mathrm{kHz}$ was obtained with a potentiostat equipped with a frequency response analyzer (Reference 600, Gamry Instruments). Weekly electrochemical impedance spectroscopy measurements of all the electrodes of the spinal implants were made in fully awake rats.

Real-time monitoring platform and control policies. The real-time monitoring and control platform was implemented within a multi-threaded C++ code (Visual Studio 2010, Microsoft) running on a quad-core Microsoft Windows 7 computer. Stimulation patterns were applied via an RZ5 processing unit (TuckerDavis Technologies) connected to an MS16 Stimulus Isolator (Tucker-Davis Technologies). The integrated Vicon recording system generated raw 3D positions of the markers, which were imported into the C++ environment in (soft) real time via Ethernet using the DataStream SDK software. We developed a custom algorithm for online interpolation of missing markers through triangulation and relabeling of each marker to the appropriate joint landmark. All signals were filtered online using least mean squares adaptive filters.

The control logic triggered individual electrodes based on trajectory of left and right hindlimb endpoints. Triggering events were extracted for each electrode individually. Control algorithms continuously calculated the angular trajectory of the foot around a virtual center of rotation in the sagittal plane. The change in on-off state of each electrode configuration was triggered when the angular values crossed user-defined thresholds that were previously optimized for each electrode and rat during mapping experiments (Supplementary Fig. 6).

The algorithm calculated for each gait-cycle $k$ the estimated position of the virtual center of rotation $\hat{\mathrm{C}}_{\mathrm{k}}$ derived from the trajectory $\mathrm{T}_{k}$ of each foot in the sagittal plane as:

$$
\left(\mathrm{x}_{\mathrm{C}}, \mathrm{y}_{\mathrm{C}}\right)=\left(x_{\max }, \frac{1}{N} \sum_{j=1}^{N} \mathrm{y}_{\mathrm{j}}\right)
$$

where $x_{\max }$ is the $x$ coordinate at the point of highest step-height $\left(y_{\max }\right)$, and $N$ the number of data points recorded per gait cycle. In order to prevent jittering owing to cycle-to-cycle variability, the estimate of the center of rotation was iteratively updated at every gait cycle as:

$$
\hat{\mathrm{C}}_{\mathrm{k}}=\hat{\mathrm{C}}_{\mathrm{k}-1}+\mu\left(C_{k}-\hat{C}_{k-1}\right)
$$

with an updating factor $\mu=0.2$. This approach captured the natural alternation of stance and swing phases during gait despite intrinsic variability within and in-between gait cycles. This procedure was also inherently invariant to scaling, and compliant enough to detect triggering-times with high fidelity compared to other methods that exhibit increase in variability with deteriorated gait movements. These control algorithms provide a flexible tool to study and implement phase-dependent modulation of neuroprosthetic system in real time during gait.

To evaluate the delay in the processing time of this loop, we simulated an input and recorded the time necessary to adapt the stimulation parameters. This delay included the processing of video recordings by the motion capture system, the transfer of this data via Ethernet to a real-time control computer, the processing of these data using dedicated libraries, the changes in the parameters of the stimulus isolator, and the delivery of the modified stimulation pulse through the plug mounted on the head of the rat. We sent concomitant pulses at opposite ends of the loop, and calculated the discrepancy between signals after each iteration for more than 100 successive cycles. The computed delays remained below 20 ms. Custom-developed C++ and Tucker-Davis Technologies codes can be made available through material transfer agreement upon request.

Behavioral recordings. Behavioral conditions. Rats were recorded under two conditions. First, bipedal locomotion was recorded on a treadmill while 
the robotic body weight support system provided optimal support against the direction of gravity and prevented lateral falls. Second, quadrupedal locomotion was recorded along a straight runway with a flat surface and along a staircase that combined a succession of 4 steps as described previously ${ }^{53}$. All the experimental conditions within and between recording sessions were randomized. Experiments were replicated at least twice on two successive days for each reported time point. Behavioral recordings were blinded after stimulation amplitudes were set by one of the experimenter, who remained with the animal and remained blinded to the stimulation conditions during experiments. A second experimenter who operated the recording equipment verified the appropriate timing of spatiotemporal neuromodulation during experiments.

Spatiotemporal neuromodulation in rats with complete SCI. Electrochemical stimulation protocols were selected on the basis of an extensive amount of previous studies in rats with complete and incomplete $\mathrm{SCI}^{5,7}$. The serotonergic replacement therapy used during training was administered systemically before testing. After a few minutes, monopolar electrical stimulation currents were delivered between relevant electrodes of the implants and an indifferent ground located subcutaneously. The intensity of electrical spinal cord stimulation was tuned $(40 \mathrm{~Hz}, 50-300 \mu \mathrm{A}$, biphasic rectangular pulses, 0.2 -ms duration) to obtain optimal stepping visually.

To optimize the timing of stimulation onset and end for each relevant electrode of the spinal implant, we performed a comprehensive mapping that linked the timing of stimulation with functional effects in rats with complete transection of the thoracic spinal cord. For each rat $(n=5)$, the duration of the gait cycle $(800 \pm 156 \mathrm{~ms}$, s.d.) was normalized then divided into 10 equal bins that served as triggering events to turn the stimulation on or off. For electrodes targeting extensor versus flexor hot spots, the stimulation was turned on or off over the entire range of bins. A total of 10 steps were usually recorded for each electrode and bin. Temporal structure was optimized on the basis of the modulation of a few gait parameters that characterized the expected effects of the stimulation. The timing of the electrodes targeting the extensor hot spot was driven by the amplitude of ankle extensor muscle activity, the amount of vertical ground reaction forces, and the intensity and amount of foot acceleration at push off (Supplementary Fig. 6). The timing of the electrodes targeting the flexor hot spot was driven by amplitude of flexor muscle activity, the amount of foot dragging, step height, and the intensity and orientation of foot acceleration at swing onset (Supplementary Fig. 6). These parameters were weighted equally for identification of the optimal temporal structure for each hot spot.

After optimization of the temporal structure, the ability of spatiotemporal neuromodulation to mediate superior facilitation of locomotion compared to continuous neuromodulation was tested in the same rats during bipedal locomotion on a treadmill. A total of 10 to 20 successive steps were recorded during continuous neuromodulation $(40 \mathrm{~Hz}, 20-200 \mu \mathrm{A}$, biphasic rectangular pulses, 0.2 -ms duration) applied through electrodes located over the midline of spinal segments L2-L3 and S1 (conventional protocols) and through spatially selective electrodes located on the lateral aspect of the same segments. Locomotor performance was compared with neuromodulation applied through the same spatially selective lateral electrodes with the identified temporal structure. The amount of robot-assisted body weight support was maintained constant across conditions. The amplitude of stimulation was adjusted for each condition. The amplitudes optimal for spatiotemporal neuromodulation could not be used for continuous neuromodulation since these values led to diminished performances, and could occasionally block hindlimb movements.

The maximum weight-bearing capacity of each rat with contusion SCI was tested under continuous neuromodulation and spatiotemporal neuromodulation around 7 weeks after SCI. From an optimal vertical weight support condition, the amount of assistance was decreased in 5\% increments after each sequence of 10 successful steps until the rat collapsed. Kinematic and muscle activity was recorded throughout these evaluations.

The endurance of rats with contusion SCI was recorded on two different days that were randomized across animals, $\sim 7$ weeks after SCI. From an optimal vertical weight support condition, rats stepped with continuous neuromodulation or spatiotemporal neuromodulation until they collapsed onto the treadmill. Concomitant kinematic recordings were used to measure the step height of each step.

Rats with contusion SCI ( $n=6)$ were evaluated during quadrupedal locomotion at 2 months after SCI, when functional recovery had plateaued ${ }^{5}$. Rats performed four successive trials without stimulation and with the serotoninergic replacement therapy and spatiotemporal neuromodulation, both along the runway and staircases. Bilateral hindlimb and trunk kinematics were recorded during these trials. The percentage of tumbles, touches, and passes over the steps was computed on the basis of video recordings. Tumbles were defined as dragging throughout the execution over the step, whereas touches characterized gait cycles during which the paw entered into contact with the steps but passed it successfully. An event was classified as 'pass' when the paw had no contact with the step during the entire swing phase.

Statistical analysis. All computed parameters were quantified and compared between tested groups unless otherwise specified. Statistics were performed on average values per rat. All data are reported as mean \pm s.e.m. unless specified otherwise. Significance was analyzed using paired Student's $t$-test, ANOVA or repeated-measures ANOVA when data were distributed normally. Post hoc comparisons were performed using the Kruskal-Wallis test. The nonparametric Mann-Whitney test was used in comparisons of $<6$ rats.

The sizes of the experimental groups were selected to minimize the number of sentient animals used in the studies while providing sufficient information to report significant and reliable results. One animal from the complete SCI group had to be excluded from the analysis of spatiotemporal maps owing to failure of some electrodes to record muscle activity. However, this animal could still be used for the analysis of whole-body kinematics, kinetics and muscle activity of ankle muscles. Gait cycles were excluded from data analysis when stimulation profiles were delivered with incorrect timing during behavioral recordings, which occurred in fewer than $3 \%$ of all gait cycles for spatiotemporal neuromodulation. For continuous neuromodulation, none of the gait cycles had to be excluded. Group sizes, methods and statistics are reported in Supplementary Table 2.

52. Minev, I.R. et al. Biomaterials. Electronic dura mater for long-term multimodal neural interfaces. Science 347, 159-163 (2015).

53. Dominici, N. et al. Versatile robotic interface to evaluate, enable and train locomotion and balance after neuromotor disorders. Nat. Med. 18, 1142-1147 (2012).

54. Stieglitz, T. \& Meyer, J.U. Implantable microsystems. Polyimide-based neuroprostheses for interfacing nerves. Med. Device Technol. 10, 28-30 (1999). 